\title{
A percepção da equipe de enfermagem na relação profissional-família da criança
}

\section{hospitalizada}

\author{
The perception of the nursing team in the professional-family relationship of the hospitalized child \\ La percepción del equipo de enfermería en la relación profesional-familiar del niño hospitalizado
}

Recebido: 03/11/2021 | Revisado: 08/11/2021 | Aceito: 09/12/2021 | Publicado: 15/12/2021

Flávia Rodrigues de Araújo Durães ORCID: https://orcid.org/0000-0002-7519-508X Faculdade Interamericana de Porto Velho, Brasil

E-mail: flaviaaraujo.rodrigues@gmail.com

Keilla dos Santos Andrade

ORCID: https://orcid.org/0000-0001-5554-4310 Faculdade Interamericana de Porto Velho, Brasil E-mail: keilla16andrade@gmail.com

Marcela Milrea Araújo Barros ORCID: https://orcid.org/0000-0002-4569-0354 Faculdade Interamericana de Porto Velho, Brasil E-mail: marcela.barros@uniron.edu.br

Vanessa de Souza Canterle ORCID: https://orcid.org/0000-0002-3891-986X Faculdade Interamericana de Porto Velho, Brasil E-mail: vanessa_canterle@hotmail.com

Andrezza Iara Rodrigues Vieira ORCID: https://orcid.org/0000-0002-7047-0871 Faculdade Interamericana de Porto Velho, Brasil E-mail: andrezza.yara@gmail.com

Bruno Gomes Brumado ORCID: https://orcid.org/0000-0001-7752-7335 Faculdade Interamericana de Porto Velho, Brasil E-mail: brunobrumado7@gmail.com

\begin{abstract}
Resumo
Objetivo: Analisar a percepção da equipe de enfermagem na relação profissional e acompanhante/familiar da criança hospitalizada em um hospital pediátrico. Metodologia: Optou-se por uma pesquisa qualitativa, descritiva com uso da técnica entrevista a partir de roteiro semiestruturado. Para análise de dados utilizou-se o método análise de conteúdo. Participaram do estudo 13 profissionais da equipe de enfermagem, dos quais cinco enfermeiros e oito técnicos de enfermagem, no período de julho a setembro de 2021. Resultados: Foram elencados dois núcleos temáticos: barreiras na relação profissional e acompanhante/familiar da criança hospitalizada e estratégias de fortalecimento para o cuidado à criança hospitalizada. De acordo com as narrativas da equipe de enfermagem, a interação, participação e o controle emocional do acompanhante são fundamentais para subsidiar a assistência, auxiliando na recuperação da criança. Foram evidenciadas ainda, a necessidade de desenvolvimento de estratégias para auxiliar na relação profissional de enfermagem e acompanhante, como suporte psicológico e qualificação profissional, bem como a atuação efetiva da equipe multiprofissional na assistência à criança e sua família, aumentando o vínculo, a comunicação e a inserção do familiar no processo de cuidar durante o período de internação. Considerações Finais: O acompanhante é parte integrante da assistência já que é o elo entre a equipe de enfermagem e o paciente pediátrico. A equipe deve estar preparada e qualificada para oferecer uma assistência humanizada, empática e resiliente que contribua significativamente para o processo de recuperação da criança.
\end{abstract}

Palavras-chave: Equipe; Enfermagem; Criança; Família.

\begin{abstract}
Objective: To analyze the perception of the nursing staff in the professional and companion/family relationship of the child hospitalized in a pediatric hospital. Methodology: We opted for a qualitative, descriptive research using the interview technique from a semi-structured script. For data analysis, the content analysis method was used. The study included 13 professionals from the nursing team, including five nurses and eight nursing technicians, from July to September 2021. Results: Two thematic nuclei were listed: barriers in the professional and companion/family relationship of the hospitalized child and strategies of strengthening for the care of hospitalized children. According to the nursing team's narratives, the interaction, participation and emotional control of the companion are essential to
\end{abstract}


subsidize care, helping the child's recovery. The need to develop strategies to assist in the professional nursing and companion relationship was also evidenced, such as psychological support and professional qualification, as well as the effective performance of the multidisciplinary team in the care of children and their families, increasing the bond, communication and the insertion of the family member in the care process during the hospitalization period. Final Considerations: The companion is an integral part of the assistance as they are the link between the nursing team and the pediatric patient. The team must be prepared and qualified to offer humanized, empathetic and resilient assistance that significantly contributes to the child's recovery process.

Keywords: Team; Nursing; Kid; Family.

\section{Resumen}

Objetivo: Analizar la percepción del personal de enfermería en la relación profesional y acompañante / familiar del niño hospitalizado en un hospital pediátrico. Metodología: Se optó por una investigación descriptiva cualitativa utilizando la técnica de entrevista a partir de un guión semiestructurado. Para el análisis de los datos se utilizó el método de análisis de contenido. El estudio incluyó a 13 profesionales del equipo de enfermería, incluyendo cinco enfermeros y ocho técnicos de enfermería, de julio a septiembre de 2021. Resultados: Se enumeraron dos núcleos temáticos: barreras en la relación profesional y compañero / familiar del niño hospitalizado y estrategias de fortalecimiento para el cuidado de los niños hospitalizados. Según las narrativas del equipo de enfermería, la interacción, participación y control emocional del acompañante son fundamentales para subsidiar el cuidado, ayudando a la recuperación del niño. También se evidenció la necesidad de desarrollar estrategias para ayudar en la relación profesional de enfermería y acompañante, como el apoyo psicológico y la calificación profesional, así como el desempeño efectivo del equipo multidisciplinario en el cuidado de los niños y sus familias, aumentando el vínculo, la comunicación y la inserción del familiar en el proceso de atención durante el período de internación. Consideraciones finales: El acompañante es parte integral del cuidado, ya que es el vínculo entre el equipo de enfermería y el paciente pediátrico. El equipo debe estar preparado y calificado para ofrecer una asistencia humanizada, empática y resiliente que contribuya significativamente al proceso de recuperación del niño.

Palabras clave: Equipo; Enfermería; Niño; Familia.

\section{Introdução}

A Política Nacional de Atenção Integral à Saúde da Criança - PNAISC implantada em 2015, foi desenvolvida com o propósito de aperfeiçoar estratégias e ações direcionadas à Saúde da Criança no território nacional, sendo orientada por princípios a fim de garantir o acompanhamento humanizado referente ao desenvolvimento da criança desde o nascimento até a adolescência, minimizando e prevenindo fatores de riscos causadores de doenças e promovendo educação em saúde abrangendo a família (PNAISC, 2018).

Os profissionais de enfermagem são responsáveis por acolher e prestar toda assistência devida, considerando os preceitos legais que envolvem a proteção integral da criança, informar o acompanhante sobre os processos que envolvem a assistência, minimizando a ansiedade e promovendo a interação com a criança e a família. A comunicação adequada gera vínculo e assim a equipe de enfermagem implementam suas atividades considerando os princípios da Política Nacional de Humanização (Santos et al., 2020).

Pela necessidade de uma assistência de enfermagem diferenciada, é indispensável que os profissionais de enfermagem avaliem como o cuidado está sendo ofertado à criança e sua família. Neste cenário, percebe-se a participação da família como instrumento significativo para a aceitação do tratamento pela criança, reduzindo assim possíveis complicações e contribuindo no processo de recuperação. O acompanhante/familiar é o maior aliado para o restabelecimento da saúde da criança sendo decisivo para esse processo (Loureiro et al., 2021).

O Estatuto da Criança e do Adolescente (ECA), em seu Art. 12 da Lei n. ${ }^{\circ}$ 8.069, de 1990, prevê a permanência em tempo integral de um dos pais ou responsável, nos casos de internação de criança ou adolescente, garantindo que o acompanhante assista os procedimentos prestados nesse período, colaborando com a equipe de enfermagem, que devidamente habilitada, atuarão na recuperação da criança.

A equipe de enfermagem no que cerne o cuidado, é a detentora do conhecimento científico especificamente, porém o acompanhante é quem vai ser o elo com a criança, sendo ele a principal fonte de captação de mudanças e variações no estado 
de saúde da mesma. É primordial que a convivência entre a equipe de enfermagem e o acompanhante se dê em plena concordância (Ribeiro et al., 2017).

A convivência entre profissional de enfermagem e acompanhante da criança hospitalizada apresenta dificuldades, pois embora reconhecendo que o profissional tenha relevância sobre a recuperação, o acompanhante acaba transferindo carga de estresse e irritabilidade para a equipe de enfermagem, tornando o profissional centro de sua insatisfação. Há ainda, comparações relacionadas aos profissionais proferidas pelos acompanhantes ocasionando indisposição na equipe (Lamb et al., 2019).

Por outro lado, frequentemente o profissional não demonstra empatia, desconsiderando as necessidades expostas pelo acompanhante/familiar, dando prioridade e agindo de forma mecânica na execução das rotinas hospitalares. As informações referentes à assistência e as normas hospitalares geralmente são passadas de forma ríspida, fato que causa no acompanhante constrangimento para expressar suas dúvidas concernentes aos cuidados e estado de saúde da criança (Silva et al., 2018).

Diante do exposto, a questão que norteia o estudo é: como o profissional de enfermagem percebe a relação acompanhante/familiar durante o período de internação da criança? Nesse sentido, o objetivo do estudo é analisar a percepção da equipe de enfermagem na relação profissional e acompanhante/familiar da criança hospitalizada em um hospital pediátrico.

\section{Metodologia}

Trata- se de um estudo descritivo e exploratório, com abordagem qualitativa, realizado em um Hospital Infantil em Porto Velho-Rondônia, região Norte do Brasil. A pesquisa qualitativa tem por objetivo observar características de determinados grupos de pessoas, fenômenos e experiências descrevendo-os sob uma determinada perspectiva, sendo essa pesquisa direcionada por instrumento, portanto, em um determinado momento realiza-se a entrevista, na qual se abordam questões inerentes ao estudo sob uma ótica singular de quem pesquisa. Posteriormente é estabelecida uma relação sobre as variáveis e a partir de então sua classificação (Salles et al., 2019; Roberto, 2017).

A pesquisa foi realizada em um pronto socorro infantil, destinado ao atendimento infantil de média e alta complexidade, pertencente à rede estadual de saúde, com 140 leitos de internação e observação. É referência para os 52 municípios do Estado e para áreas fronteiriças (Estados do Amazonas, Mato Grosso e Acre e a República da Bolívia). Os serviços médicos hospitalares são oferecidos nas diversas especialidades, que vão do diagnóstico à terapêutica, através de procedimentos de assistência ao tratamento clínico e cirúrgico e ainda o atendimento a egressos dando continuidade do atendimento/acompanhamento de forma ambulatorial (CONASS, 2017).

Neste estudo definem-se como população, os profissionais da equipe de enfermagem, especificamente enfermeiros e técnicos de enfermagem do hospital. Como critério de seleção amostral, optou-se pela conveniência, considerando os profissionais de enfermagem que exerciam sua atividade laboral nos setores de internação, e ainda que se dispusesse a participar voluntariamente do estudo, constituindo a seguinte amostra final: 05 (cinco) enfermeiros e 8 (oito) técnicos de enfermagem, totalizando 13 (treze) profissionais de enfermagem.

Foram considerados critérios de inclusão para a realização da pesquisa: ser profissional de enfermagem, enfermeiro ou técnico de enfermagem, com experiência profissional de no mínimo dois (2) anos na pediatria, profissionais que atuavam diretamente no setor de internação do referido hospital, integrantes do quadro permanente de servidor do referido hospital, estando disposto a ser entrevistado seguindo roteiro semiestruturado proposto de livre e espontânea vontade. Como critérios de exclusão: os profissionais que atuavam nos setores de emergência e unidade de terapia intensiva pediátrica, por serem locais com peculiaridades próprias, bem como os profissionais que estavam de férias e licença no período da coleta de dados. 
Para coleta de dados, o primeiro contato foi reservado para apresentação dos objetivos do estudo e o caráter voluntário na participação. Diante do aceite foi oferecido o Termo de Consentimento Livre e Esclarecido (TCLE) para leitura individual, ficando evidente a liberdade para o mesmo desistir de participar da pesquisa a qualquer instante. A entrevista foi agendada com o profissional de enfermagem de acordo com sua disponibilidade sem, portanto interferir no processo assistencial. Cada entrevista durou aproximadamente 15 minutos, em ambiente e o horário de escolha de cada participante. A entrevista contou com um roteiro semiestruturado de cinco perguntas que foram gravadas em dispositivo de áudio e após a análise, as gravações foram descartadas.

Para a análise dos dados, empregou-se a análise de conteúdo conforme proposto por Minayo (2012), o qual é constituído por 3 fases: pré-análise: as gravações foram transcritas na íntegra e organizadas de forma ordenada, a fim de favorecer a observação primária dos dados; exploração do material: os relatos foram separados e agrupados por afinidade de respostas, formando conjuntos (matriz de síntese) para classificação do material em categorias empíricas de análise e; tratamento dos resultados: para inferência e interpretação dos dados analisados, tornou-se necessário realizar releitura e reorganização do material de forma sintetizada, porém preservando o valor informações. Os resultados foram confrontados com a literatura pertinente a fim de promover reflexão ao tema abordado.

Para garantir o sigilo e a privacidade dos dados e o anonimato dos envolvidos, as falas dos participantes foram transcritas na íntegra, e nos resultados apresentados utilizou-se a codificação sequencial por categoria profissional e ordem de participação na entrevista (Enfermeiros - Enf1, Enf2, Enf3, Técnicos de Enfermagem - Tec1, Tec2, Tec3, por exemplo).

O estudo foi aprovado pelo Comitê de Ética em Pesquisa (CEP), da UNIÃO EDUCACIONAL DO NORTE UNINORTE, sob $\mathrm{n}^{\circ}$ de parecer 4.480.068, CAAE: 40739920.0.0000.8028 em consideração aos princípios éticos concernentes às pesquisas que envolvem seres humanos, considerando a Resolução n 466, de 12 de dezembro de 2012 do Conselho Nacional de Saúde/Ministério da Saúde.

\section{Resultados e Discussões}

A amostra da pesquisa foi constituída por 13 profissionais da enfermagem (Enfermeiros e Técnicos de enfermagem), atuantes nos setores de internação do hospital infantil. Destes, 05 são enfermeiros, dos quais 04 do sexo feminino e 01 do sexo masculino, com média de idade de 44 anos e tempo de serviço em pediatria nos limites de 8 a 35 anos, com média de 15 anos. Dos 08 técnicos de enfermagem, 07 foram do sexo feminino e 01 do sexo masculino, com média de idade de 37,5 anos e tempo de serviço em pediatria entre os limites de 04 e 20 anos e média de 9 anos, conforme exposto no Quadro 1.

Quadro 1 - Caracterização dos participantes enfermeiros e técnicos de enfermagem do hospital infantil de Porto Velho, RO, 2021.

\begin{tabular}{|c|c|c|c|}
\hline Entrevistados & Idade & Sexo & $\begin{array}{c}\text { Tempo de Serviço em } \\
\text { Pediatria }\end{array}$ \\
\hline ENF 1 & 60 anos & Feminino & 35 anos \\
\hline ENF 2 & 55 anos & Masculino & 25 anos \\
\hline ENF 3 & 36 anos & Feminino & 8 anos \\
\hline ENF 4 & 44 anos & Feminino & 15 anos \\
\hline ENF 5 & 36 anos & Feminino & 20 anos \\
\hline TEC 1 & 44 anos & Feminino & 18 anos \\
\hline TEC 2 & 43 anos & Feminino & \\
\hline
\end{tabular}


Research, Society and Development, v. 10, n. 16, e436101624307, 2021

(CC BY 4.0) | ISSN 2525-3409 | DOI: http://dx.doi.org/10.33448/rsd-v10i16.24307

\begin{tabular}{|c|c|c|c|}
\hline TEC 3 & 50 anos & Feminino & 15 anos \\
\hline TEC 4 & 52 anos & Masculino & 12 anos \\
\hline TEC 5 & 28 anos & Feminino & 6 anos \\
\hline TEC 6 & 32 anos & Feminino & 4 anos \\
\hline TEC 7 & 28 anos & Feminino & 4 anos \\
\hline TEC 8 & 30 anos & Feminino & 4 anos \\
\hline
\end{tabular}

Fonte: Durães et al. (2021).

$\mathrm{Na}$ análise empírica dos dados, tendo como base relatos semelhantes e convergentes, a partir da matriz de síntese, fora elencados dois núcleos temáticos: Barreiras na relação profissional e acompanhantelfamiliar da criança hospitalizada e Estratégias de fortalecimento para o cuidado à criança hospitalizada.

\subsection{Barreiras na relação profissional e acompanhante/familiar da criança hospitalizada}

A hospitalização é uma situação estressante tanto para a criança quanto para o acompanhante, que na maioria das vezes é a genitora. Além de deixar sua função profissional, de cônjuge e cuidador da família, o acompanhante também precisa enfrentar grandes mudanças no cotidiano, como administrar os sentimentos em relação à doença e ao tratamento, tendo que se adaptar à nova rotina hospitalar com o filho doente. Nesse novo universo emergem emoções como irritabilidade, tristeza, medo e impotência, resultantes do fato de estar no ambiente hospitalar, que constantemente é visto como insensível impessoal e aterrorizante. Isso não é uma escolha, mas uma necessidade, em uma atmosfera de expectativa e medo (Minssen et al., 2021).

O sentimento de medo resultante do processo de internação da criança, é constantemente partilhado pelo acompanhante de forma negativa com os profissionais de enfermagem durante a relação estabelecida. $\mathrm{O}$ medo acaba criando uma situação estressante e a tendência da criança e seu acompanhante é relacionar aos profissionais de enfermagem os momentos de tensão, resultante dos procedimentos realizados, que geralmente provocam desconforto e até mesmo dor (Azevedo, 2017). Segundo as narrativas da equipe, percebe-se que o ambiente hospitalar apresenta-se como hostil e o medo, dúvidas e inseguranças são sentidos pelo acompanhante:

"Eu percebo assim, que ele chega com muito medo do hospital e com muitas dúvidas em relação à patologia das crianças". (ENF4)

"[...] o hospital parece muito comum e normal para o profissional, mas para o acompanhante e especialmente para criança não deixa de ser um ambiente hostil". (ENF 2).

"Os pais chegam com uma carga muito pesada de medo, de insegurança, pois desconhecem os procedimentos que está sendo realizados no seu filho". (TEC1).

O medo, a insegurança e as dúvidas são sentimentos que permeiam os acompanhantes. Tais sentimentos evidenciados pelos profissionais de enfermagem podem interferir diretamente no tratamento da criança hospitalizada. A Política Nacional de Humanização (PNH) prevê a inclusão do usurário nos processos de administração e de cuidado, para que este participe da assistência e também contribua com propostas para melhoria do sistema e do cuidado. A inclusão visa o incentivo e a criação de formas inovadoras de prestar o cuidado e estruturar o serviço. Cabe ao profissional de enfermagem na unidade de internação pediátrica, envolver e requerer a cooperação da família, sensibilizando-os que o tratamento da criança vai além das medicações administradas. A equipe de enfermagem deve apoiar acompanhante a enfrentar suas preocupações, incentivando-os a buscar soluções e a continuarem no desenvolvimento de seus cuidados (Macedo et al., 2017). 
Considerando a relevância do envolvimento do acompanhante no cuidado da criança internada, o qual é reconhecido como essencial para atender às necessidades e manutenção do bem-estar do paciente, diversos estudos mostram os benefícios em ter famílias envolvidas no processo de cuidar, no qual é imprescindível que os profissionais de enfermagem se empenhem em integrar o acompanhante no processo de tratamento da criança (Martins, 2020).

Segundo Salgado et al. (2018), quando os profissionais de enfermagem veem o acompanhante como um indivíduo que interfere negativamente na assistência prestada, não cooperativo e resistente, essa situação possivelmente está relacionada às dificuldades do acompanhante em entender e enfrentar o processo de adoecimento e hospitalização da criança. Percebeu-se no estudo, que os conflitos entre equipe de enfermagem e acompanhante ocorriam com frequência durante a execução de determinados procedimentos, como por exemplo, durante uma punção venosa, havendo muita resistência por parte do acompanhante. Tal atitude além de não favorecer o sucesso do procedimento, cria uma atmosfera de tensão e aflição, causadas pela superproteção da criança.

Tais dificuldades também foram relatas pelos profissionais de enfermagem, conforme observamos nas falas a seguir:

"[...] o acompanhante não tem essa consciência do quanto é importante o acesso para criança e tem a 'furada' como algo ruim para o paciente, acabam preferindo que o filho fique sem acesso [...] porque eles têm muita resistência à coisas simples". (TEC8)

"Ter um acompanhante que não colabora, que não aceita as orientações e não ajuda no tratamento, contribui para que a criança tenha uma qualidade de assistência limitada". (ENF4)

"[...] se o cuidador não colaborar, não vai ter uma assistência adequada”. (ENF3)

Diante da dificuldade da compreensão e assimilação do acompanhante com resistência e pouca colaboração no desenvolvimento do cuidado, é necessário que os profissionais de enfermagem exercitem a paciência, empatia e resiliência, para lidar com o acompanhante nessas situações, comunicando-se de forma clara e concisa, expondo as verdades e detalhes dos procedimentos a serem realizados, certificando-se que o acompanhante entendeu o que foi orientado.

O relacionamento adequado e harmonioso entre acompanhante e os diversos profissionais de saúde atuantes no hospital, é fundamental para um atendimento humano e de qualidade, tornando-se imprescindível para que a prestação de serviço evolua conforme preconizado pela PNH, no qual a comunicação é a ferramenta principal de ligação entre profissionais de enfermagem, criança e acompanhante. A comunicação inadequada resulta na fragilidade da confiança entre as partes envolvidas, comprometendo o sentimento de segurança em que o acompanhante desenvolveria, tornando-se uma relação pouco atrativa, não alcançando assim a atenção da criança e seu responsável (Schliemann et al., 2021).

De acordo com Hoffmann et al., (2019), a deficiência na comunicação está relacionada em mais de 70\% dos eventos adversos ocorridos durante assistência, ocasionando prejuízo ao paciente, aumentando o período de internação e os gastos com o tratamento excedente. A comunicação efetiva, geralmente é percebida pelo acompanhante como uma vulnerabilidade do sistema, tornando-se necessárias ações de qualificações profissionais. Neste sentido, os profissionais expõem situações em suas narrativas às dificuldades enfrentadas concernentes as falhas de comunicação.

"[...] exames que são marcados em longo prazo e a mãe não é informada sobre a data prevista [...] a mãe fica agoniada esperando, sendo que já está marcado o procedimento, mas o acompanhante não foi comunicado a respeito". (TEC6)

“[...] o próprio profissional que por sua vez não está preparado para escutar a mãe, temos que saber escutar”. (TEC3) 
"[...] só o fato de ouvir e tentar acalmar e traçar algumas soluções, já ajuda a reduzir sua ansiedade, mas nem todo profissional tem essa estratégia ou essa paciência ao se comunicar com o acompanhante”. (ENF 5)

Observa-se que a comunicação entre profissional de saúde e acompanhante não é eficiente, pois o profissional nem sempre está disposto a dar a devida atenção ao acompanhante. Tal atitude faz com que o acompanhante se retraia e muitas vezes guarde dúvidas sobre procedimentos, doença e tratamento da sua criança, fato este que contribui inclusive para uma resistência à determinados procedimentos.

É de suma importância que os profissionais tenham entendimento sobre a melhor forma de abordar o acompanhante, buscando sempre a cordialidade e a empatia. A comunicação segundo Hoffmann et al., (2019), deve ser adequada conforme o nível de instrução de cada indivíduo, buscando sempre sanar as dúvidas e sempre apresentar a importância dos procedimentos a serem executados e da sua participação no tratamento do filho.

A comunicação é uma condição básica que permite a interação e promove o contato entre as pessoas, pois subsidia a troca de conhecimentos, gestos e emoções. Através da comunicação, muitas informações e sentimentos podem ser passados de uma pessoa para outra, percebendo o entendimento ou desenvolvimento entre as partes (Salgado, et al., 2018).

\subsection{Estratégias de fortalecimento para o cuidado à criança hospitalizada.}

\section{Atuação em equipe multiprofissional e interdisciplinar}

Para o acolhimento necessário e o bom desempenho da equipe no geral, é muito importante que o acompanhante ao ser acolhido obtenha as informações adequadas, de forma limpa e clara, para que possa processar de maneira correta a informação transmitida, considerando inclusive os motivos particulares que o levaram até ali, pois esse acompanhante pode estar chegando com uma sobrecarga de processos emocionais e pessoais que podem trazer ainda mais ansiedade e insegurança diante do novo cenário (Boeger, 2020; Pascoal \& Souza, 2021).

Em alguns relatos, vamos perceber que a atenção da equipe de enfermagem no que cerne ao trabalho em equipe multiprofissional, como por exemplo, a psicologia e o serviço social, entre outros é percebida de forma positiva, constituindo este, um eixo que aproxima a relação entre o acompanhante e a equipe, além de torna mais leve esta relação. A narrativa evidencia:

“Acho que as mães deveriam ter um acompanhamento de algum psicólogo, ou ter algo que as ajudassem”- (TEC5).

"Eu percebo que o acompanhante, principalmente a mãe, quer atenção, então às vezes ela tá ali e precisa somente ser ouvida" - (ENF5).

“[...] A assistência social poderia intervir, porque uma das brigas maiores que a gente tem com o acompanhante é na hora de dormir [...]” (TEC7).

Pelas narrativas acima, observa-se a necessidade de se estreitar as atuações profissionais de maneira interdisciplinar em prol de um cuidado holístico e integralizado à criança hospitalizada e sua família. A percepção dos problemas internos será melhor identificada para os profissionais que trabalham em colaboração, compartilhando as necessidades individualmente.

Se o trabalho não for integralizado, cada profissional avaliará a criança de forma objetiva e subjetiva. Tal estratégia permite abordagens diferentes para problemas específicos e subsidiam a escolher a terapia mais adequada e consequentemente, há melhoria na qualidade da assistência com prognósticos mais satisfatórios.

É inegável que todo paciente precisa de assistência, devendo esta ser prestada preferencialmente por equipe multidisciplinar, sendo o enfermeiro o profissional que no período de hospitalização está mais presente desde o início até a alta, estabelecendo relação com o paciente e consequentemente com o seu acompanhante e familiares. Portanto é indispensável 
à interação harmoniosa dessa tríade, principalmente quando é levado em consideração o paciente pediátrico (Fassarella et al., 2019).

O trabalho em equipe multiprofissional e interdisciplinar, do ponto de vista integral, tem se mostrado um desafio para a gestão dos serviços de saúde, e tem se manifestado como um nó-crítico que exige dos profissionais uma relação de benefício mútuo, participação, responsabilidade compartilhada e solidariedade, que cada um entenda os pensamentos, opiniões e desejos uns dos outros e promova exercícios que ainda são difíceis para a maioria dos profissionais que não estão habituados a compartilhar decisões (Martins et al., 2018).

Nesta perspectiva, cada profissional deve refletir sobre o seu papel frente às atividades desenvolvidas. De acordo com as falas, observa-se que esta temática é relevante para a equipe de enfermagem, pois, um trabalho desarticulado, pode sobrecarregar os demais profissionais:

“Cada profissional deve desempenhar seu papel, para não sobrecarregar o profissional enfermeiro" - (ENF4).

"Eu acredito que cada um fazendo sua parte, ajuda muito, principalmente em dar atenção" (ENF 5).

A sobrecarga é um conceito multidimensional que envolve o campo do biopsicossocial, que surge do equilíbrio entre o tempo disponível e os cuidados prestados, as condições físicas, sociais e psicológicas, e a atribuição e distribuição de funções. Seja na qualidade de vida dos trabalhadores ou na qualidade da assistência, é um fator preocupante, com implicações para uma assistência desumanizada, insatisfação e falta de motivação profissional (Santos et al., 2020).

"[...] como trabalhamos com uma demanda muito exaustiva nem sempre conseguimos dar atenção ao acompanhante $"($ TEC5).

"[...] as demandas de trabalho são extensas e cada dia vem aumentando, então tem muita cobrança do acompanhante em relação ao enfermeiro e a enfermagem [...]". (ENF4)

A sobrecarga de trabalho suprime do profissional, momentos de interação e atenção às necessidades intrínsecas ao acompanhante e paciente, atendo-se apenas ao tratamento, agindo de forma mecanizada. A relação positiva entre paciente, acompanhante e profissional, será a base para elo de confiança que esses indivíduos formaram. Desse modo, o processo de enfermagem deve ser norteado pela dimensão interpessoal, unificando tecnologia, ciência e a individualidade, proporcionado um ambiente humanizado, potencializando a produtividade saudável. (Santos et al., 2020).

Os relatos evidenciam neste contexto, a preocupação da equipe de enfermagem com os cuidados sobre as informações transmitidas, a necessidade das demais profissões estarem à disposição para orientar essas famílias, uso de protocolos gerais ou algo que contribua para a elucidação das dúvidas e aproximá-los ainda mais da assistência.

A estruturação do processo de trabalho pressupõem que há normas e práticas que proporcionam o convívio harmônico entre os diversos profissionais, pacientes e familiares no ambiente hospitalar, visando assistir à criança com qualidade e pacificamente, de preferência sem resistências e conflitos, favorecendo o conforto e assim contribuindo diretamente na recuperação e manutenção da saúde e bem-estar na pediatria (Fassarella et al., 2019).

Portanto, estima-se que o ambiente hospitalar pediátrico deve ter profissionais de saúde, incluindo os de enfermagem, sensíveis a fatores psicológicos, não apenas da criança, mas também do seu acompanhante, de modo que suas ações não se limitem apenas a seu físico, tratamento e ou procedimento, sendo necessária dos profissionais de enfermagem uma relação que promova o vínculo de confiança na criança e no seu acompanhante (Paula et al.,2019). 


\section{Cuidar de quem cuida das crianças hospitalizadas e sua família}

Tendo em vista o distanciamento do meio social e das atividades cotidianas, a hospitalização infantil é considerada um evento estressante e traumático para as crianças, acarretando grandes mudanças em seus hábitos diários. Deve-se observar que essas mudanças incluem restrições, novas rotinas, pessoas desconhecidas e procedimentos que causam desconforto, medo e / ou dor e que podem refletir diretamente naquele que cuida o profissional de enfermagem (Paula et al., 2019).

$\mathrm{Na}$ análise dos relatos, observa-se que os profissionais vivenciam situações em que controle, preparo emocional, resiliência e empatia fazem parte das suas rotinas, sendo crucial para a assistência expondo assim, a necessidade de acompanhamento de um profissional psicólogo para cuidar de quem cuida da criança hospitalizada e sua família, como podemos observar nas narrativas:

"[...] cada pessoa tem um comportamento diferente, educação e cultura diferente, então é muito difícil lidar com tudo isso." (TEC4).

"Se tivesse um atendimento psicológico para o profissional, melhoraria muito, pois lidar com o ser humano não é uma questão fácil." (ENF1).

"Eu não sabia muito como lidar, tinha medo de falar com o acompanhante”(TEC7).

"Eu particularmente não me sinto psicologicamente preparada para fazer um atendimento a uma criança com acompanhante, porque a gente não está preparada para eles" (TEC8).

A equipe de enfermagem compõe a categoria profissional com maior desgaste emocional, devido à convivência com os pacientes e os constantes processos de internações, sendo as situações que eles vivenciam no seu dia a dia de trabalho (o sofrimento, a dor, a doença), um dos fatores que mais contribuem para tal, ter a oportunidade de encontrar um espaço para falar livremente sobre as tensões e dificuldades encontradas no cotidiano hospitalar, suas emoções com pessoas que vivenciam as mesmas situações as fazem sentir - se ouvidas, compreendidas e apoiadas por profissionais que focam não só no paciente, mas também na família e na equipe que acompanha o processo (Silvestri et al., 2021).

Quando um profissional não consegue se adaptar aos estressores do ambiente de trabalho e não desenvolve estratégias de enfrentamento eficazes, ficará em estado de exaustão emocional e sujeito a transtornos mentais, principalmente a síndrome de Burnout, que está intimamente relacionada a este, pela prática profissional decorrente das relações interpessoais e organizacionais (Barros \& Gonçalves, 2019).

Ter um conhecimento prévio da área de atuação é uma forma de se preparar para os diversos casos que a equipe vê diariamente. Nesse contexto, a forma de lidar com as emoç̃es e as relações a criança e sua família pode compreender melhor o assunto e tornar mais transparente a transmissão e a acessibilidade das pessoas que vivenciam tais eventos (Silvestri et al., 2021).

\section{Disseminação da Política Nacional de Humanização}

A atitude da equipe de enfermagem para com as famílias da criança hospitalizada é a base da qualidade do cuidado. Nesse sentido, o enfermeiro como líder da equipe de enfermagem, deve reconhecer a importância do envolvimento e participação da família nos cuidados que prestam ao filho durante a internação. As ações de enfermagem devem ter como objetivo orientar as famílias para que possam participar ativamente do cuidado aos filhos (Martins, 2020).

Considera-se a família uma parte frágil e pouco confusa quando o assunto e informação é o conhecimento do processo assistencial, principalmente quando se trata de um hospital pediátrico. O profissional de enfermagem deve entender que o 
hospital não é seu espaço, procurando adaptar o acompanhante a este novo ambiente, ajudando-a na organização do seu cotidiano para lidar com o impacto da hospitalização da criança (Macedo et al.,2017).

Os profissionais entrevistados, de maneira empírica, referem algumas estratégias para desempenhar uma assistência humanizada, porém como vemos nas falas abaixo, o conhecimento da Política Nacional de Humanização ainda é muito limitada:

"Olha já ouvi falar, mas assim detalhadamente, seria interessante eu saber um pouco mais dessa politica" - (ENF5).

"Conheço muito pouco, quase nada [...] os problemas vão aparecendo e a gente vai tentando solucionar" - (ENF1).

"É a primeira vez que estou ouvindo falar nessa política" - (TEC3).

É importante compreender e valorizar os profissionais de enfermagem e incorporá-los na tomada de decisões, e investir na sua capacidade de analisar, definir e validar processos de trabalho, visto que a valorização do trabalhador e a cogestão são umas das diretrizes da Política Nacional de Humanização (PNH, 2015).

Os usuários do SUS devem compreender que a humanização da saúde busca valorizar os diferentes sujeitos envolvidos no processo de saúde, entre os quais funcionários, usuários e gestores do SUS, o objetivo é formar uma rede de participação, que evidencie se ocorrem mudanças na atenção ao usuário e gerenciamento de fluxo de trabalho. É extremamente importante que as diretrizes do HumanizaSUS sejam totalmente aplicáveis em um ambiente onde são prestados cuidados de saúde (Sousa et al., 2021).

Os valores do cuidado humanizado, como na produção saudável, valorizam e incentivam os usuários. A forma de evidenciar esse tipo de humanização é a ação: desde o acolhimento da equipe de saúde, a promoção da confiança e o contato com os usuários, o ouvinte que é qualificado pode escolher pessoas prioritárias, vulneráveis, graves e de risco. A PNH é uma ferramenta importante e básica do Sistema Único de Saúde, pois visa aprimorar e divulgar os planos humanizados existentes, além de implementar processos de monitoramento e avaliação para evidenciar os que vêm sendo gerados no SUS (Palheta et al., 2020).

Uma assistência qualificada é essencial para a sistematização e capacitação da equipe de enfermagem. A capacitação e qualificação dos profissionais de enfermagem é essencial, pois são eles os principais responsáveis por manter a unidade funcionando 24 horas por dia, todos os dias do ano (Santiago \& Medeiro, 2020).

Neste sentido, a assistência em pediatria conduz à um âmbito de reflexão que pode proporcionar a sensibilização do profissional de enfermagem a fim de que este perceba a individualidade de cada paciente, considerando que por se tratar de uma criança há um grau maior de dificuldade da família e paciente em lidar com o processo de adoecimento. Receber a criança e seu acompanhante adequadamente no ambiente hospitalar ameniza sensação de medo e ansiedade, favorecendo o estabelecimento de uma relação saudável, contribuindo para melhora da criança e tornando efetiva a prática da humanização na assistência pediátrica (Strefling et.al., 2018).

\section{Considerações Finais}

Ainda há muito que se avançar no que diz respeito aos fatores humanos relacionados ao cuidado e a relação equipe de enfermagem e cuidador familiar, tendo em vista que tais aspectos são subjetivos. Avalia-se a necessidade de uma comunicação efetiva, de apoio psicológico tanto do profissional quanto do acompanhante e atuação de equipe multiprofissional, pois as informações referentes à rotina e procedimentos ficam somente a cargo da enfermagem. 
Considera-se que o acompanhante é parte integrante da assistência e de fundamental importância, já que ele é o elo entre a equipe de enfermagem e o paciente pediátrico. A equipe deve estar preparada e qualificada para oferecer uma assistência humanizada, empática e resiliente que contribua significativamente para o processo de recuperação da criança.

\section{Referências}

Barros, K. G. G., \& Gonçalves, J. R. (2019). Aspectos psicológicos que envolvem os cuidados paliativos pediátricos. Revista JRG de Estudos Acadêmicos, 2(5), 156-165.

Biasibetti, C., Hoffmann, L. M., Rodrigues, F. A., Wegner, W., \& Rocha, P. K. (2019). Comunicação para a segurança do paciente em internações pediátricas. Revista Gaúcha de Enfermagem, 40

Boeger, M. (2020). Hotelaria hospitalar: gestão em hospitalidade e humanização. Editora Senac São Paulo.

Araújo Minssen, M. E., da Silva, F. P., \& de Oliveira, M. G. C. (2021). "Acompanhante também precisa de acompanhante": Reflexões sobre a rotina das mães em uma Enfermaria Pediátrica Cardiológica. Research, Society and Development, 10(7), e37110716604-e37110716604.

Sousa, J. E. N., Resende, A. S. S., de Carvalho Falcão, S. M. A., de Sousa, M. L., Silva, M. P. B., de Carvalho, N. C., \& Santiago, R. F. (2021). Humanização na assistência hospitalar: estudo teórico-reflexivo. Revista de Casos e Consultoria, 12(1), e25446-e25446.

Santos Azevêdo, A. V., Júnior, A. C. L., \& Crepaldi, M. A.(2017). Interação equipe de enfermagem, família, e criança hospitalizada: revisão integrativa.

Fassarella, B. P. A., Ribeiro, W. A., Freitas, L. D. M., Do Nascimento, J. C., dos Santos, J. C. C., \& da Fonseca, C. D. S. G. (2019). Equipe de enfermagem x acompanhante na pediatria: o impacto dessa parceria na assistencia pediatrica. Nursing (São Paulo), 22(258), 3319-3324.

Lamb, F. A., Beck, C. L. C., Coelho, A. P. F., \& Vasconcelos, R. O. (2019). Trabalho de enfermagem em pronto socorro pediátrico: entre o prazer e o sofrimento. Cogitare enferm, 24, e59396.

Lei $\mathrm{n}^{\mathrm{o}}$ 8.069, de 13 de julho de 1990. Dispõe sobre o Estatuto da Criança e do Adolescente e dá outras providências. http://www.planalto.gov.br/ccivil_03/leis/18069.htm.

Loureiro, F. M., Antunes, A. V. D. R. A., \& Charepe, Z. B. (2021). Theoretical nursing conceptions in hospitalized child care: scoping review. Revista Brasileira de Enfermagem, 74.

Macedo, I. F. D., Souza, T. V. D., Oliveira, I. C. D. S., Cibreiros, S. A., Morais, R. D. C. M. D., \& Vieira, R. F. C. (2017). As concepções da equipe de enfermagem frente à família da criança hospitalizada. Revista Brasileira de Enfermagem, 70, 904-911.

Martins, Celestina de Barros. (2020). Envolvimento e Participação das Famílias nos Cuidados à Criança Hospitalizada: Atitudes dos Enfermeiros na ilha de Santiago em Cabo Verde. https://docs.bvsalud.org/biblioref/2021/04/1177516/d2019_10002322116_21723001_2.pdf0

Martins, P. L., Azevedo, C. D. S., \& Afonso, S. B. C. (2018). O papel da família nos planos de tratamento e no cuidado pediátrico hospitalar em condições crônicas complexas de saúde. Saúde e Sociedade, 27, 1218-1229.

Minayo, M. C. D. S. (2012). Análise qualitativa: teoria, passos e fidedignidade. Ciência \& saúde coletiva, 17, 621-626.

Palheta, R. P., \& da Silva Lima, A. C. (2020). A humanização em saúde visão dos usuários de um hospital público. Brazilian Journal of Health Review, 3(5), $14553-14565$.

Pascoal, M. M., \& de Souza, V. (2021). A inserção da hotelaria nos hospitais públicos. Revista Ibero-Americana de Humanidades, Ciências e Educação, 7(6), 231-244.

Paula, G. K. D., Góes, F. G. B., Silva, A. C. S. S. D., Moraes, J. R. M. M. D., Silva, L. F. D., \& Silva, M. D. A. (2019). Estratégias lúdicas no cuidado de enfermagem à criança hospitalizada. Rev. enferm. UFPE on line, 1-11.

Política Nacional de Atenção Integral à Saúde da Criança (PNAISC) no âmbito do Sistema Único de Saúde (SUS). http://biblioteca.cofen.gov.br/wpcontent/uploads/2018/07/Pol\%C3\%ADtica-Nacional-de-Aten\%C3\%A7\%C3\%A3o-Integral-a-Saude-da-Crian\%C3\%A7a.pdf.

Política Nacional de Humanização $\quad$ de HumanizaSUS, https://bvsms.saude.gov.br/bvs/folder/politica_nacional_humanizacao_pnh_1ed.pdf.

Relatório de Gestão (2017) Evolução da saúde em Rondônia: https://www.conass.org.br/RAG-ESTADOS/RO_Relatorio_Gestao_Anual_2017.pdf Ribeiro, J. P., Gomes, G. C., Thofehrn, M. B., Mota, M. S., Cardoso, L. S., \& Cecagno, S. (2017). Criança hospitalizada: perspectivas para o cuidado compartilhado entre enfermagem e família. Rev. enferm. UFSM, 1-13.

Roberto, GFPFKMGA Metodologia Científica e Gestão de Projetos 2017. [Digite o Local da Editora]; Editora Saraiva, 2017.9788536531533. https://integrada.minhabiblioteca.com.br/\#/books/9788536531533/.

Salgado, M. A., Bittencourt, I. S., Salgado, M. A., do Nascimento Paixão, G. P., Marinho, C. L. A., \& de Sena Fraga, C. D. (2018). Percepção da enfermagem acerca do acompanhante no cuidado à criança hospitalizada. Ciência \& Saúde, 11(3), 143-150.

Salles, A. L. D. O., Sampaio, C. E. P., Pereira, L. D. S., Malheiros, N. S., \& Gonçalves, R. A. (2019). O enfermeiro e a questão da adesão do paciente ao tratamento da hipertensão arterial sistêmica. Rev. enferm. UERJ, e37193-e37193. 
Research, Society and Development, v. 10, n. 16, e436101624307, 2021

(CC BY 4.0) | ISSN 2525-3409 | DOI: http://dx.doi.org/10.33448/rsd-v10i16.24307

Santiago, E. R., \& Medeiros, V. H. (2020). Ambiente lúdico na assistência pediátrica em pacientes hospitalizados: uma ressignificação no atendimento.

Santos, C. D. S. C. S., Abreu, D. P. G., de Mello, M. C. V. A., da Silva Roque, T., \& Perim, L. F. (2020). Avaliação da sobrecarga de trabalho na equipe de enfermagem e o impacto na qualidade da assistência. Research, Society and Development, 9(5), e94953201-e94953201.

Santos, K. C. O. D., Oliveira, I. C. D. S., Martinez, E. A., Azevedo, M. D. S. N., Carmo, S. A. D., \& Dias, R. A. B. (2020). Informações transmitidas às famílias das crianças na emergência na perspectiva da Enfermagem. Rev. Pesqui.(Univ. Fed. Estado Rio J., Online), 1087-1092.

Schliemann, A. L., Razza, L. C., da Silva, M. A., Lima, P. P., \& Catão, T. P. (2021). Desenvolvimento de material que facilite a convivência e a comunicação em enfermaria pediátrica. Brazilian Journal of Development, 7(1), 2816-2828.

Silva, M. C. F. C. R. D., Borba, R. I. H. D., Onishi, J. Y. T., Horta, A. L. D. M., \& Ribeiro, C. A. (2018). Interação do técnico em enfermagem com a família do paciente hospitalizado. Acta Paulista de Enfermagem, 31, 359-365.

Silvestri, A. P. S., dos Santos, E. L., Belini, G. F., Pellizzaro, A. O., da Silva Moura, T., Gonçalves, S., ... \& Moi, A. (2021). Equipe de enfermagem frente aos cuidados paliativos em pediatria: Revisão integrativa. Research, Society and Development, 10(5), e13910514848-e13910514848.

Strefling, I., Ribeiro, J. P., Gomes, G. C., Klemtz, F. V., Oliveira, B. B., \& Lopes, K. B. (2018). Potencialidades da ambiência para a humanização da unidade de pediatria. Revista da Jornada de Pós-Graduação e Pesquisa-Congrega Urcamp, 510-522. 